\title{
Asia desde la academia Sudamericana
}

\section{Asia from the South American academy}

Santiago Carranco Paredes

Universidad Internacional del Ecuador, Ecuador

Emilia Ruiz Revelo

Pontificia Universidad Católica del Ecuador, Ecuador

Autor para correspondencia: scarrancoparedes@gmail.com, emiliajaelruiz@gmail.com Fecha de recepción: 23 de mayo de 2018 - Fecha de aceptación: 22 septiembre de 2018

\section{Resumen}

La idea de que Asia y los estudios sobre este continente deben tener una gran preeminencia para la academia sudamericana y noroccidental se ha convertido en un cliché. Los estudios que han confirmado la preponderancia de estados asiáticos para la comprensión de las relaciones comerciales, sociales, políticas, económicas y académicas actuales, tanto a un nivel global como regional, son vastos. El presente estudio no se va a preocupar por evidenciar la expansión de la influencia asiática dentro de la academia sudamericana, más bien, se limitará a reseñar el modo en el que las universidades Sudamericanas están estudiando al sudeste asiático por medio de un estudio cuantitativo que percibió como variables principales las materias sobre Asia en la región, sus contenidos y las universidades que las dictan.

Palabras Clave: Asia; América del Sur; academia; integración

\begin{abstract}
The idea that Asia and the studies of this continent should have a great preeminence for the South American and Northwestern academies has become a cliché. Several studies has confirmed the preponderance of Asian states for the understanding of current commercial, social, political, economic and academic relations. The present study is not going to worry about showing the expansion of the Asian influence within the South American academy, rather, it will be limited to review the way in which the South American universities are studying Southeast Asia through a quantitative study that perceived as main variables, the subjects that study Asia in the region, their contents and the universities that dictate them.
\end{abstract}

Key Words: Asia; South America; academy; integration 


\section{Introducción}

Dentro del marco de las $\mathrm{V}$ jornadas de Relaciones Internacionales, celebradas en la ciudad de Buenos Aires en noviembre del 2017, se trató un tema importante relacionado con la manera en la que se percibe y se aporta a la disciplina de las Relaciones Internacionales desde el Sur, especialmente desde la academia sudamericana. Una de las conferencias magistrales del evento estuvo a cargo de Amitav Acharya, quien expuso parte de su último trabajo, en el cual expresó la necesidad imperante de la universalización de la disciplina, es decir que no exista una ruptura entre la producción académica noroccidental, con las que están afuera de la mencionada esfera de influencia, como la asiática, africana o Sudamericana. En su texto, Acharya (2014), propone una disciplina global, cambiar la percepción de Relaciones Internacionales occidentales o relaciones internacionales del Sur por algo más amplio e inclusivo que son las Relaciones Globales.

Se ha visto que, desde la década de los ochenta, se ha producido una desconcentración del monopolio del conocimiento científico a nivel global, la importancia de la academia no occidental en las Estudios Internacionales ha tomado fuerza sobre todo desde la caída del muro de Berlín, ya que ninguna teoría noroccidental pudo explicar de manera integral este proceso. Por otra parte, la sociedad internacional se ha visto afectada por un desplazamiento continuo del centro del poder geopolítico desde el Noroccidente hacia la región de Asia-Pacífico; un cambio que, si en un primer momento fue económico, progresivamente se ha ido convirtiendo también en político y cultural.

De igual manera, se ha vislumbrado que los mercados emergentes asiáticos, especialmente los del sudeste, han ido ascendiendo en la cadena de valor de los procesos de producción industrial. Para El Aynaoui y Woertz 2016, este proceso de industrialización, no solo ha impactado en los indicadores económicos que son expresados en las fluctuaciones de los mercados globales, sino que ha afectado el desarrollo propio de las sociedades occidentales.

Es así como el sudeste asiático, ha sido capaz de desarrollar una especie de "Soft Power" lo que en un sentido se lo concibe como la forma de influencia sutil de la cultura o de las ideas, frente a formas más coercitivas de ejercer presión, también llamadas poder duro, como por ejemplo la acción militar, o como las presiones y condicionamientos de tipo económico (Nye 2009). De esta forma se recalca cierta creciente influencia cultural asiática en el occidente, ya sea en el ámbito de la cultura pop, la filosofía, el mundo académico o la arquitectura; así lo demuestra el éxito del conocido "Gangnam Style", las improvisadas salas de yoga en los gimnasios tradicionales, la ola de inauguraciones de restaurantes de cocina asiática, los diseños urbanos futuristas y la creación de un ranking propio de las universidades del mundo (ARWU, por sus siglas en inglés) elaborado por la Universidad Jiao Tong de Shanghái, en donde existen más de 100 universidades del sudeste asiático dentro del top500 a nivel mundial (ShanghaiRanking Consultansy 2016).

La idea de que Asia y los estudios sobre este continente deben tener una gran preeminencia para la academia Sudamericana y noroccidental se ha convertido en un cliché. Los estudios que han confirmado la preponderancia de estados asiáticos como China, Japón, Corea del Sur, India entre otros para la comprensión de las relaciones comerciales, sociales, políticas, económicas y académicas actuales, tanto a un nivel global como regional, son vastos. De igual 
manera, la evidencia empírica confirma el papel preponderante que representa el sudeste asiático en la configuración de la sociedad internacional contemporánea, por lo que en la actualidad se hace inconcebible en el estudio académico de Relaciones Internacionales dejar de lado al continente asiático (Kohno 2014) (Bisley 2011).

En la última década, la academia Sudamericana exhibió una fascinación por el crecimiento de China y su creciente influencia en esta región. Un número considerable de autores latinoamericanos se dedicó a entender la dinámica de estas nacientes relaciones entre ambos continentes, entre ellos (Manríquez \& Álvarez) (2014) y Panizza (2008), entre otros. Esto motivó a que exista una gran producción regional sobre el tema, lo cual llevó a que varias editoriales regionales dediquen espacios exclusivos para artículos relacionados con China o con el sudeste asiático. Un ejemplo de esto es el caso de la Revista Andina de Estudios Políticos que en 2016 publicó un Dossier titulado "China y América Latina: industrias extractivas, política exterior e integración". Por su parte, la Revista Estudios de la Universidad de Costa Rica publicó en el mismo año un Dossier exclusivamente para tratar "El Estudio sobre China desde América del Sur"'. Por otra parte, un tema predominante en el último semestre ha sido el de incluir los saberes no occidentales al mainstream de las Relaciones Internacionales, justamente como lo propone Acharya, es por esta razón que un sinnúmero de congresos, jornadas y eventos de relaciones internacionales de la región Sudamericana están concentrados en legitimar estos saberes que vienen desde afuera de la influencia occidental. Sin duda, estas épocas de un cambio de paradigma en las ideas podrán marcar un punto de partida para entender una materia inclusiva de relaciones internacionales.

\section{Metodología}

El presente estudio no se enfocó en evidenciar la expansión de la influencia asiática dentro de la academia sudamericana, más bien, se limitará a reseñar el modo en el que las universidades de la región están estudiando al sudeste asiático. Para el estudio se ejecutó un análisis estadístico de codependencia de variables cualitativas a partir de una muestra de las mejores universidades de cada región, obtenidas del Ranking Web de Universidades

(Webometric). De este ranking se tomó a las 64 mejores universidades de Sudamérica ${ }^{1}$, a cada una de ellas se les atribuyó un número de variables relacionadas con el estudio de Asia en el campo de las Relaciones Internacionales, ya que como se lo mencionó previamente, esta carrera tendría la necesidad de afrontar académicamente el fenómeno de la expansión de la influencia asiática hacia el mundo occidental. Para el análisis de las variables se utilizó el estadístico de correspondencia simple de variables, el cual permite evaluar la relación de dependencia entre las variables seleccionadas. Es así como se logró llegar a una percepción general sobre la manera en la que la academia Sudamericana está incluyendo el estudio de Asia dentro de las mallas curriculares de la disciplina de las Relaciones Internacionales.

Adicionalmente, se analizó la percepción de los estudiantes sudamericanos sobre Asia, por medio de entrevistas estructuradas $(\mathrm{N}=7)$ que contenían una serie, de entre 12 a 14 preguntas, dirigidas a resaltar las diferencias metodológicas y empíricas que existen entre la academia Sudamericana y asiática. Por lo que se eligió una muestra de estudiantes de las Carreras de

\footnotetext{
${ }^{1}$ Los datos de las universidades venezolanas se tomaron de páginas privadas y de universidades que disponen de información, al encontrar que las páginas de universidades públicas tienen una restricción electrónica internacional.
} 
Relaciones Internacionales de las universidades de la Región Andina y Sudamericana que cuenten con dos características principales, la primera es que hayan cursado una materia relacionada con Asia en su pregrado, y la segunda que hayan realizado algún tipo de curso académico en la región del Sudeste asiático. De esta manera, se pudo definir no sólo la percepción general de la situación de las universidades respecto a su estudio de Asia, sino también el tipo de información que se difunde dentro de las clases relacionadas con dicho continente.

El análisis de la muestra se realizó en función de las principales universidades de los países sudamericanos. A su vez, se establecieron categorías sobre las materias relacionadas con Asia con el objetivo de homologar materias similares bajo una misma variable, reduciendo el número de materias a cuatro ítems: Estudios Asiáticos, Estudios Afroasiáticos, Relaciones Internacionales de Asia y Asia-Pacífico. En los gráficos que se presentan a continuación se puede apreciar estas distinciones.

\section{Principales Resultados}

Después del análisis realizado a la información recopilada se observa que, de la muestra de universidades que se utilizaron en el presente estudio, el $44 \%$ imparten la disciplina de Relaciones Internacionales. Al analizar por países, se observa que Brasil es quien cuenta con el mayor número de universidades que dentro de sus ofertas se encuentra esta carrera (39\%); seguido por Ecuador con el $21 \%$. Por su parte, Perú es un país que no presenta universidades que oferten la carrera de Relaciones Internacionales.

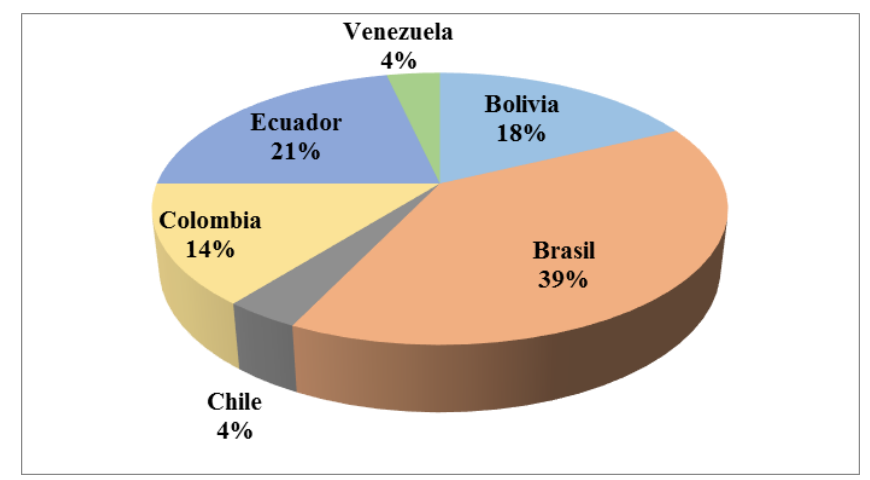

Gráfico 1: Porcentaje de Universidades que ofrecen la carrera de Relaciones Internacionales. (Gráfico Elaborado Por el Autor)

La importancia de estudiar Relaciones Internacionales viene dada en parte por la globalización que ha vivido el mundo en los últimos años. Esto ha desenvocado en que cada vez sea más relevante conocer cuáles son los actores que intervienen en las relaciones entre estados, a fin de identificar el comportamiento de cada uno de ellos y su influencia en el desarrollo de los países. Tal como se visualiza en el gráfico superior, en función de los países analizados, Brasil es el país que en mayor medida oferta la carrera de Relaciones Internacionales, esto evidencia la importancia que dicho país le da a los estudios de política internacional. El resultado tiene coherencia si consideramos que Brasil es el país de Sudamérica con mayor economía, 1.8 trillones en el 2017 (World Economic Forum 2017). 
$\mathrm{Al}$ analizar las materias que se imparten en las universidades de Sudamérica, se puede observar en el Gráfico 2, que el estudio de relaciones internacionales está presente en el $43 \%$ de universidades de la región. Se evidencia que el $20 \%$ de universidades de la muestra tienen materias específicas sobre Asia, incluyendo materias relacionadas con Asia Pacífico y Relaciones Internacionales de Asia.

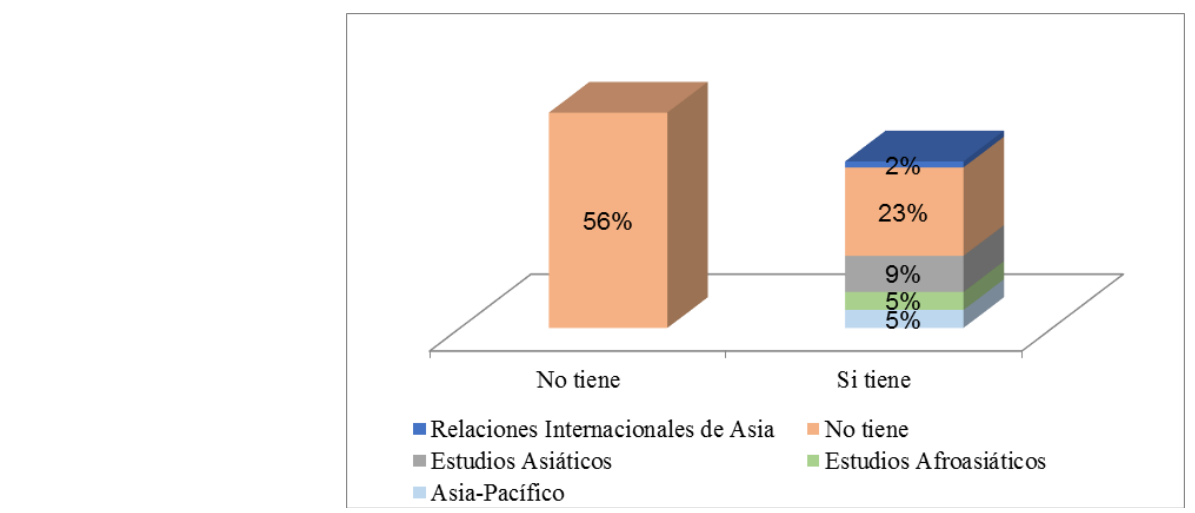

Gráfico 2: Porcentaje de Universidades que ofrecen la Carrera de Relaciones Internacionales en Sudamérica (Gráfico Elaborado Por el Autor)

La importancia de Asia para el mundo es incuestionable, es claro que este ha pasado de ser el continente místico y desconocido para el occidente, como lo proponía Edward Sid (BeitHallahmi 1980), a ser un objeto de estudio de la academia occidental. Por otro lado, Asia ha despertado y ahora se ha transformando en el productor de conocimiento. El ranking de universidades de Shanghái es una prueba de la fragmentación y de la autonomía que propone la academia asiática hacia la región occidental.

Cuando se observa a nivel de país (Gráfico 3), se evidencia que Brasil, Colombia, Ecuador y Chile son los países que dentro de sus universidades presentan materias relacionadas con estudios enfocados en Asia. La materia que más importancia presenta en la región es Estudios Asiáticos. Adicionalmente, se puede observar que Perú y Venezuela, son países que no imparten materias con relación al continente asiático.

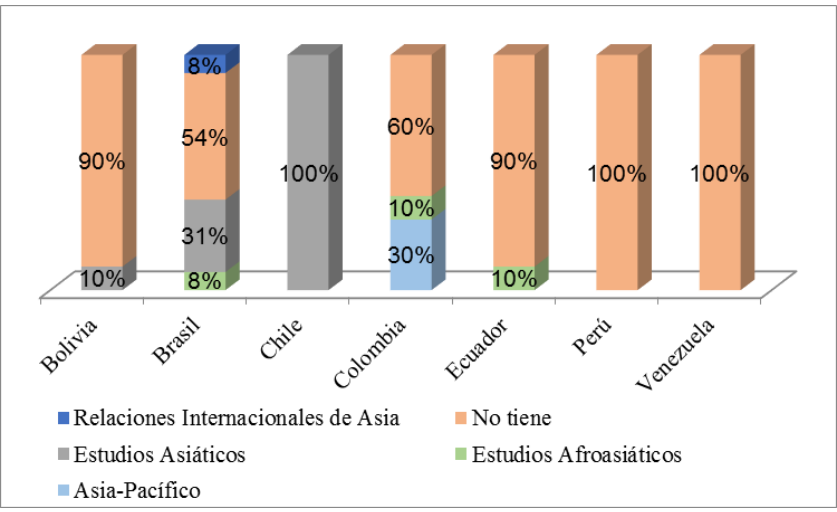

Gráfico 3: Porcentaje de Materias de Relaciones Internacionales que ofrecen la Universidades enfocadas en Asia por país de origen

(Gráfico Elaborado Por el Autor) 
Hu-DeHart (2010), explica que durante la década de los 80 y 90 la academia brasilera, argentina y chilena se enfocó en estudiar los flujos migratorios que se dieron desde Asia y el Medio Oriente hacia América del Sur luego de la segunda guerra mundial, lo cual originó una especie de costumbre académica multicultural. Adicionalmente, era común ver estudiantes de origen asiático cursando sus estudios especialmente en universidades brasileras y argentinas, por lo que el interés en los estudios asiáticos se fue ganando un espacio dentro de la academia en esta región (Ko 2016). En este sentido, se puede concebir que el estudio sobre flujos migratorios es lo que catapultó a los estudios asiáticos a tener un papel importante dentro de esta región, es claro que siendo un tema concerniente a Política Internacional sea justamente las escuelas de Relaciones Internacionales la que lo aborden.

El Gráfico 4, el cual es concerniente a un análisis de las universidades mejor ubicadas en el ranking Wbometric de universidades latinas que tienen la carrera de Relaciones Internacionales, evidencia que las universidades de la región presentan parcialmente importancia hacia los estudios asiáticos dentro de la materia de relaciones internacionales, siendo Brasil el país que más revisa los estudios referentes a Asia.

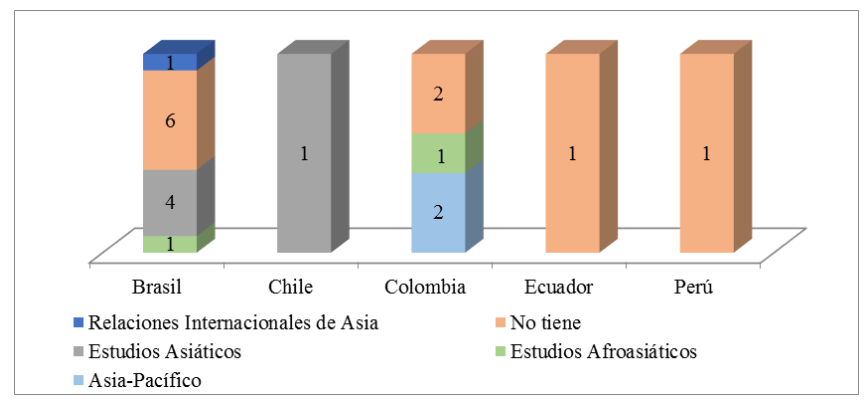

Gráfico 4: Número de Universidades con la carrera de Relaciones Internacionales enfocada en Asia, por país en base a las 20 mejores universidades del ranking latinoamericano.

(Gráfico Elaborado Por el Autor)

Si bien en los últimos años se ha dado importancia a la academia asiática desde Sudamérica, aún es bajo el número de universidades que ya han implementado materias relacionadas con dicho continente. Esto puede estar dado por las diferencias que la academia sudamericana y asiática presenta. Cuando se le preguntó a una estudiante que realizó sus estudios en la Universidad de Pekín, ella manifestó que algunas características como la disciplina, la pertinencia y la responsabilidad en el estudio de determinadas áreas marcan la diferencia entre el mundo occidental y oriental, si bien son características principales de los países tecnológicos, son aplicadas también en los países con menor grado de investigación.

En lo que respecta a la región Andina se vislumbra que existe un bajo porcentaje de escuelas de Relaciones Internacionales, a su vez, el interés sobre estudios asiáticos aún no ha despertado, algo que es corroborado al observar las respuestas de los alumnos andinos que han migrado a Asia. Exponiendo una síntesis de sus respuestas se puede deducir que su interés sobre los estudios asiáticos no nace en su pregrado, sino que este nace desde la necesidad profesional. 


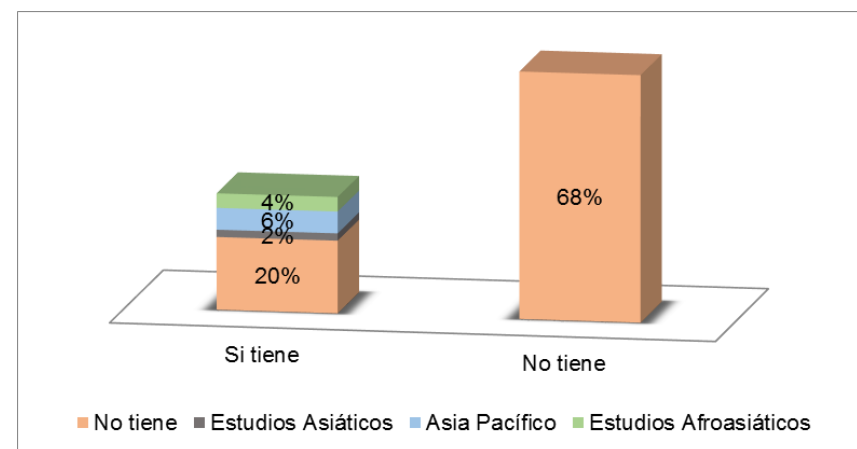

Gráfico 5: Porcentaje de Universidades que ofrecen la carrera de Relaciones Internacionales en la Región Andina. (Gráfico Elaborado Por el Autor)

Cuando se le preguntó a una estudiante andina que cursa su maestría en la Universidad Politécnica de Hong Kong nos indicó que "El sistema educativo es uno de los contrastes más significativos entre América latina y Asia. La metodología y el pensamiento de la academia oriental, limitándome específicamente a China, están muy fundamentados en la doctrina de Confucio. En tal sentido, el enfoque siempre será de una tendencia colectivista en el cual prevalecen los intereses globales sobre los particulares."

La presencia de los estados asiáticos, en especial de China en el territorio Andino, se ha extendido en los últimos años, y queda claro que existe un vacío dentro de los estudiantes que salen de la academia sobre las cuestiones referentes a Asia, por lo que los estudiantes tratan de llenarlo a partir de una búsqueda ya sea de maestrías o cursos de verano en universidades asiáticas.

Dentro de la información obtenida de algunas entrevistas, se pudo evidenciar que, si bien se ha dado mayor fuerza al análisis de estudios de Asia en Sudamérica, especialmente por la relación económica y política que las dos regiones presentan, el apoyo interno para promover los estudios asiáticos es mínimo, y principalmente está dado por conferencias promovidas por los representantes diplomáticos.

$\mathrm{Al}$ analizar las universidades del Ecuador, se obtiene que solo una universidad cuenta con disciplinas que hacen referencia a los estudios asiáticos, la cual es la Universidad Internacional del Ecuador (UIDE). Cuando se le preguntó a la profesora Natalia Encalada, quien es la que lleva dicha materia, respondió que ser la única universidad ecuatoriana enfocada en estos temas es fruto de la visión que se tiene de la importancia de Asia para el continente sudamericano, además hizo énfasis que los procesos de integración asiáticos son diferentes a los nuestros por lo que es un tema importante para estudiarlo en relaciones internacionales. 


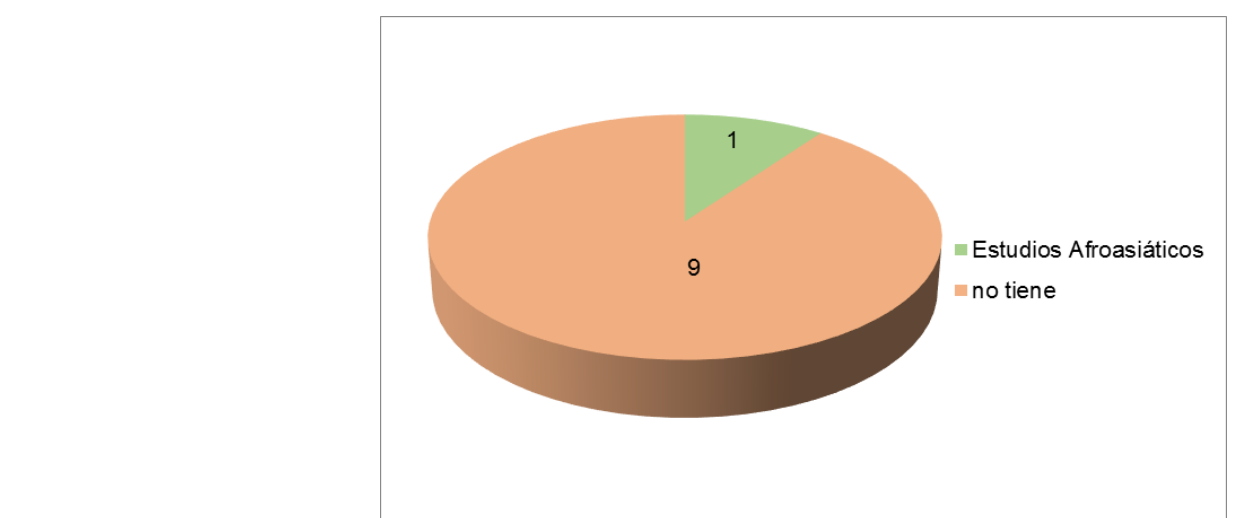

Gráfico 6: Porcentaje de materias relacionadas con los Estudios Asiáticos en las Universidades del Ecuador que ofertan la carrera de Relaciones Internacionales

(Gráfico Elaborado Por el Autor)

\section{Discusión}

Una de las razones que ha influenciado en la relación entre Asia y Sudamérica, está determinada por la complementariedad que estas dos regiones tienen. Por un lado, Sudamérica es productor de materias primas, mientras que Asia es especializada en producción manufacturera con alto grado tecnológico. Por ejemplo, durante los años de la expansión económica de China, los países sudamericanos fueron proveedores de commodities, lo cual generó una estrecha relación entre las dos partes. Como menciona (Santiso 2006) la estrategia de expansión exterior generalizada por parte de las multinacionales emergentes chinas también fue un parámetro que permitió que Asia sea considerada como una de las principales regiones importadoras de productos sudamericanos. Adicionalmente, la relación no está sólo dada a nivel económico, sino también está ligada a las relaciones políticas. China mantiene asociaciones estratégicas con varios países sudamericanos: Brasil, Venezuela, Chile, Ecuador, Colombia, entre otros.

Como señala (Rubiolo 2015) los cambios en los últimos quince años en las estructuras de demanda, la complementariedad de las matrices productivas exportadoras, y el crecimiento de las regiones, son algunos de los principales elementos que han permitido que Sudamérica presente una relación más fuerte con el continente asiático. Este interregionalismo que presentan las dos regiones, en parte está dada por su complementariedad.

Una estudiante ecuatoriana que siguió una serie de cursos de especialización en Corea del Sur, comentó que una de las motivaciones de enfocarse en los estudios asiáticos está dada por las múltiples lecturas que existen sobre los modelos económicos utilizados en esta región, y el deseo de conocer sobre las políticas aplicadas que les han permitido llegar a conocerse como potencias mundiales.

Es importante destacar que el rápido crecimiento económico de China e India, y el desarrollo que se ha producido en los estados del Sudeste Asiático han atraído la atención de académicos e investigadores, y ha tenido un gran impacto en el campo de los estudios asiáticos. Esto a su vez ha producido un cambio en la percepción que se tiene de Asia no como un objeto de estudio, sino como un productor de conocimiento (Winichakul 2014). 


\section{Conclusiones}

Luego del término de la Guerra Fría, la academia tuvo que exponerse a varios procesos de cambio para poder adaptarse a esta nueva era de globalización dentro del sistema unipolar. Por esta razón, en los últimos años la idea de la internacionalización de la academia se ha convertido en un tema preponderante para las universidades a nivel mundial. Este pensamiento de la internacionalización ha abierto un campo interesante para que estudiantes occidentales tengan acceso al conocimiento de Asia. Después del análisis realizado, y los resultados expuestos, se observa que Sudamérica presenta una relación económica y política con el continente asiático, sin embargo, en el sector académico se está iniciando la institucionalización de materias relacionadas con el Asia, especialmente en las relaciones internacionales. No obstante, Ecuador en especial, al igual que otros estados de la región andina, sufre un retraso en cuanto a la incorporación de estudios relacionados con Asia en sus mallas curriculares, por lo que los estudiantes interesados en Asia han tenido que recurrir a vías alternas para poder acceder a dichas esferas de conocimiento.

\section{Bibliografía}

Acharya, Amitav (2014) the End of American World Order. Edition: 1. Cambridge Malden, MA: Polity Press.

Beit-Hallahmi, Benjamin (1980) Review of Orientalism. Journal for the Scientific Study of Religion 19(1): 69-70.

Bisley, Nick (2011) Asia's Transformation, International Relations and Public Policy. Australian Journal of International Affairs 65(1): 102-108.

Durkin, Kathy (2008) The Middle Way: East Asian Master's Students' Perceptions of Critical Argumentation in U.K. Universities. Jornal of Studies in International Education 12(1): $38-55$.

El Aynaoui, Karim y Eckart, Woertz (2016) Introducción: África, América Latina y El siglo de Asia. Revista CIDOB d'afers Internationals 114(1): 7-16.

Hu-DeHart, Evelyn (2010) Multiculturalism in Latin American Studies: Locating the "Asian" Immigrant; or, Where Are the Chinos and Turcos? Latin American Research Review 44(2): 235-242.

Ko, Chisu Teresa (2016) Toward Asian Argentine Studies. Latin American Research Review 51(4): 271-289.

Kohno, Masaru (2014) East Asia and International Relations Theory. International Relations of the Asia-Pacific 14(1): 179-190.

Manríquez, J. \& Álvarez, L (2014) Mao's steps in Monroe's backyard: towards a United StatesChina hegemonic struggle in Latin America? Revista Brasileira de Política Internacional 8(1): 9-27. 
Nye Jr, Joseph (2009)Soft Power: The Means to Success In World Politics. Hachette UK.

Panizza, Francisco (2009) Contemporary Latin America Development and Democracy Beyond the Washington Consensus. New York: Zed Books.

Rubiolo, Florencia (2015) Las relaciones entre el Sudeste asiático y América del Sur: el interregionalismo como complemento de las vinculaciones bilaterales. CIDOB d'Afers Internacionals 113(1): 211-234

Santiso, Javier 2006. ¿Realismo mágico? China e India en América Latina y África. Economía Exterior 38 (3): 60-69 http://www.oecd.org/dev/38447180.pdf

ShanghaiRanking Consultansy (2016) Discovering World Class. https://doc-0k-aodocs.googleusercontent.com/docs/securesc/kffmvot8g48hsb9807ci97cds8c3tqu0/hj9v9d6 jid5eu5k3ead8q2o4oq561533/1496419200000/08619350468429608082/08334199373923 561037/0Bw2rAawlHlvBSFZiVnBNZUFCVDA?e=download\&nonce=8bcm $891 \mathrm{da} 9 \mathrm{t} 4 \mathrm{~s} \&$ user $=08334199373923561037 \&$ hash=ihka660f0spm9samua3qbc2opnjfhieb, accessed June 2, 2017.

Winichakul, Thongchai (2014) Asian Studies across Academies. The Journal of Asian Studies 73(04): 879-897. 